\title{
Fabrication of Periodic 3D Nanostructuration for Optical Surfaces by Holographic Two-Photon-Polymerization
}

\author{
Yi-Hsiung Lee, Yi-Jui Liu, Patrice L. Baldeck, and Chih-Lang Lin
}

\begin{abstract}
This study fabricated periodic 3D nanostructures by using a periodic voxel array for light extraction. Two-photon polymerization (TPP) is a well-known technology used for generating complex 3D micro- and nanostructures with sub-100-nm resolution. Because of the periodic voxel-by-voxel array fabrication process, the fabrication of holographic two-photon polymerization (HTPP) can be faster than that of traditional TPP. HTPP promises a flexible technique to fabricate an arbitrary periodic 3D structured optical surface. In this research, we used a $25 \times 25$ beam-splitting holographic mask to generate a periodic focus in a photocured material and to systematically fabricate complex 3D nanostructures at rates of $\mathbf{~ m m}^{2}$ per minute. The optical responses of nanostructures with a $1 \mu \mathrm{m}$ period fabricated in a polymer were characterized using 3D optical microscopy. The experimental results of the light distribution demonstrated favorable agreement with those of the simulation. In the light-extracting experiment, the light extraction capability of the gold-layer nanostructures was 3.6 times higher than that of the glass without nanostructures.
\end{abstract}

Index Terms-Two-photon polymerization, HTPP, nanostructures, electroless gold plating, light distribution, light extraction.

\section{INTRODUCTION}

In the past decade, periodic polymer nanostructures have been extensively used in light applications such as optical data storage [1], fiber optics [2], and photonic crystals [3]. Periodic polymer nanostructures can be generated using rolling-mask lithography [4], laser interference lithography [5], and laser direct writing [6]. Laser-direct writing can be used to fabricate arbitrary 3D micro- and nanostructures easily by applying two principles: subtraction-type and addition-type [7]. Subtraction-type laser direct writing, Cheng et al. [8] employed femtosecond laser to directly generate micro- and nanostructures onto the ITO surface of LEDs but generated debris and thermal damage. Addition-type laser direct writing, also called two-photon polymerization (TPP) fabrication, is the potential technology for fabricating an arbitrary 3D nanostructure, which is integrated with many voxels [9], [10]. A voxel is generated in regions in which the laser energy is higher than the threshold energy by using an

Manuscript received August 25, 2015; revised March 20, 2016.

Yi-Hsiung Lee is with the Ph.D. Program of Electrical and Communications Engineering, Feng Chia University, Taichung 407, Taiwan (e-mail: andy955305@gmail.com).

Yi-Jui Liu is with Department of Automatic Control Engineering, Feng Chia University, Taichung 407, Taiwan (e-mail: erliu@ @fcu.edu.tw).

Patrice L. Baldeck is with University Grenoble, I/CNRS, LIPhy UMR 5588, Grenoble, F-38041, France (e-mail: baldeck@ujf-grenoble.fr).

Chih-Lang Lin is with Institute of Biomedical Engineering and Materials Science, Central Taiwan University of Science and Technology, Taichung 406, Taiwan (e-mail: cllin101943@ ctust.edu.tw). objective lens in a photocured material without debris and thermal damage [11].

TPP technology has several advantages, such as no need for a special operating environment, a sub-100-nm resolution, and simple fabrication of a 3D complex structure by using diverse types of photocured materials. Conventional TPP manufacturing is not effective in fabricating high-porosity or thin structures at fabrication rates ranging from $\mathrm{mm}^{2}$ to $\mathrm{cm}^{2}$, because of voxel-by-voxel fabrication processing. Therefore, to shorten the processing time, a TPP system can be integrated with particular instruments such as microlenses [12], scanners [13], and holographic masks [14]. Holographic two-photon polymerization (HTPP) fabrication is a common method that involves using a holographic mask to separate a single beam to multiple foci. In the fabrication methods, multiple foci are periodically fabricated along voxels arranged in a parallel configuration in a photocured material. The processing time is shortened because of the parallel configuration of the voxels, which enable a simultaneous voxel-by-voxel processing. In other words, HTPP promises a flexible technique to fabricate arbitrary periodic 3D micro/nanostructure by means of point-by-point processing.

In this study, we employed HTPP to fabricate a 3D structured optical surface. We used a $25 \times 25$ beam-splitting hologram with a TPP system to simultaneously fabricate arbitrary periodic 3D nanostructures at a rate of $\mathrm{mm}^{2}$ per minute. Periodic polymer nanostructures were used for light distribution experiments and periodic polymer nanostructures with gold layers were used for light extraction experiments.

\section{EXPERIMENT METHODS}

\section{A. Design}

For enhancing light exaction, we sought to design a structure that can be integrated with five voxels (Fig. 1(a)). A single nanostructure was integrated with five voxels: The base layer was integrated with four voxels and the second layer was integrated with a single voxel. The distance between voxels was $170 \mathrm{~nm}$ in the $\mathrm{x}$ - or $\mathrm{y}$ direction, and the layer thickness was $600 \mathrm{~nm}$ in the $\mathrm{z}$ direction. Fig. 1(b) shows a $25 \times 25$ array of the integrated voxels. The pitch between nanostructures was $1000 \mathrm{~nm}$.

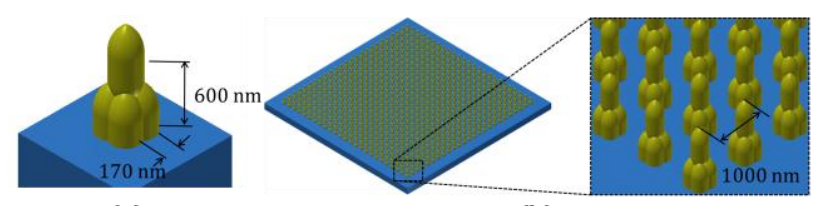

(a)

(b)

Fig. 1. (a) Dimension of a nanostructure, and (b) dimension of $25 \times 25$ nanostructures and their details. 


\section{B. Instruments}

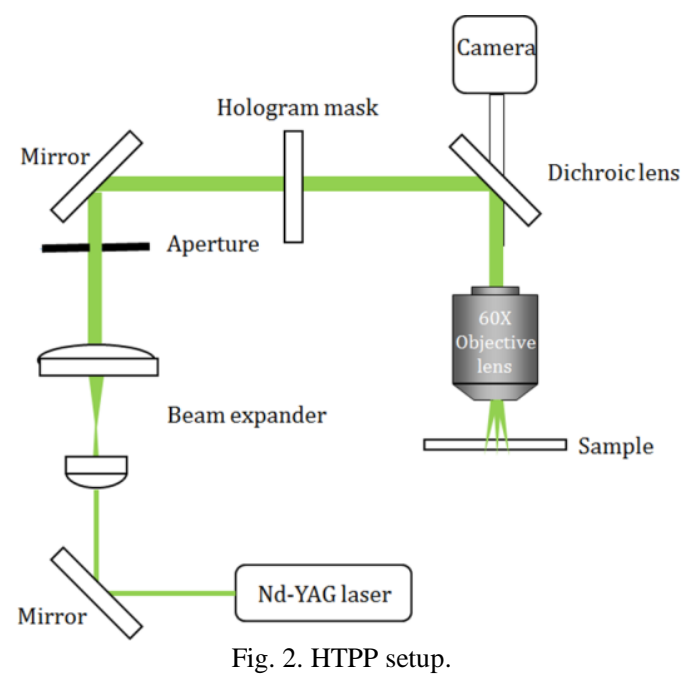

As shown in Fig. 2, the fabrication was performed using 1-ns pulses from an amplified self-Q-switched Nd-YAG laser with a second-harmonic wavelength at $532 \mathrm{~nm}$, a repetition rate of $135 \mathrm{kHz}$, and an average power of $358 \mathrm{~mW}$ (each beamlet was $0.57 \mathrm{~mW}$ ). The expanded laser beam was phase-modulated by a transmission hologram immediately before focalization in a $25 \times 25$ array of foci by using a $60 x$ oil-immersion objective lens with a numerical aperture of 1.45. Arbitrary 3D nanostructures can be produced by moving the XYZ piezo stage according to computer-aided design trajectories. For HTPP fabrication, the photocured material used was PEGDA, and the reflective index was 1.47 according to the SIGMA company datasheet. After HTPP fabrication, the sample was immersed in 4-methy1-2-pentanone for $5 \mathrm{~min}$ and acetone for $5 \mathrm{~min}$, and was subsequently dried for the light distribution experiment. For the light extracting experiment, we immersed the sample in a $20 \% \mathrm{v} / \mathrm{v}$ solution of ethylenediamine in absolute ethanol for $5 \mathrm{~min}$ before drying and immersing the sample in absolute ethanol for $10 \mathrm{~min}$ and deionized (DI) water for $10 \mathrm{~min}$. The sample was then immersed in a $0.1 \%$ wt $\mathrm{HAuCl}_{4}$ aqueous solution for $20 \mathrm{~min}$ and DI water for $10 \mathrm{~min}$, and was subsequently dried for obtaining periodic nanostructures with gold layers.

\section{Fabrications}

For nanostructure fabrication, we integrated a holographic mask with the TPP system to separate $25 \times 25$ multiple foci from a laser beam on the image plane of the $60 x$ oil-objective lens; thus, we could fabricate 625 voxels simultaneously in the photocured material. The distance between voxels was $1000 \mathrm{~nm}$ because the distance between laser foci was $1000 \mathrm{~nm}$ In HTPP fabrication, the 625 voxels were fabricated at the 10 $\mathrm{ms}$ exposure time and $10 \mathrm{~ms}$ delay time, after which the piezo stage was moved according to CAD design trajectories for the next voxels fabrication. The total HTPP fabrication time of the $25 \times 25$ periodic nanostructures was $100 \mathrm{~ms}$.

\section{Measurements}

After HTPP fabrication, the nanostructures were characterized using scanning electron microscopy (SEM), and their optical responses were investigated using a bright-field $3 \mathrm{D}$ microscope (the scanning range above the glass surface was $5 \mu \mathrm{m}$ with a $0.2-\mu \mathrm{m}$ interval and that below the glass surface was $5 \mu \mathrm{m}$ with $0.2-\mu \mathrm{m}$ interval). The light distribution experimental results were compared with those of the simulation of light distribution, which involved conducting COMSOL simulation. For the light extraction experiment, white light was excited from the lamp and passed through the 325-385-nm bandpass filter. The light was then reflected using a dichroic lens, passed through a 20x objective lens, and illuminated the gold-layer nanostructures. After light extraction, the extraction light passed through a $20 \mathrm{x}$ objective lens, the dichroic lens, and then through a 400-nm long pass filter. The light intensity signal was obtained using an optical fiber and spectrometer after applying a long-pass filter. Fig. 3 shows this optical setup.

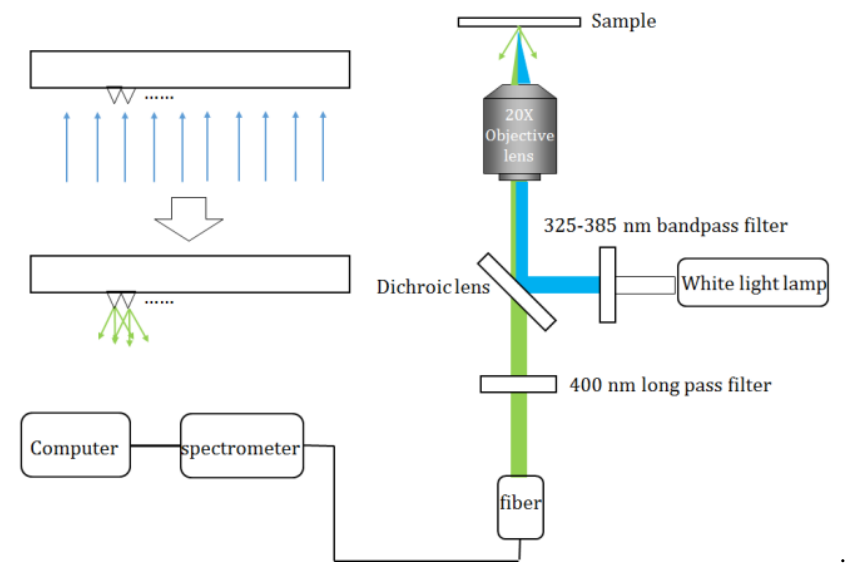

Fig. 3. Optical setup of light extraction.

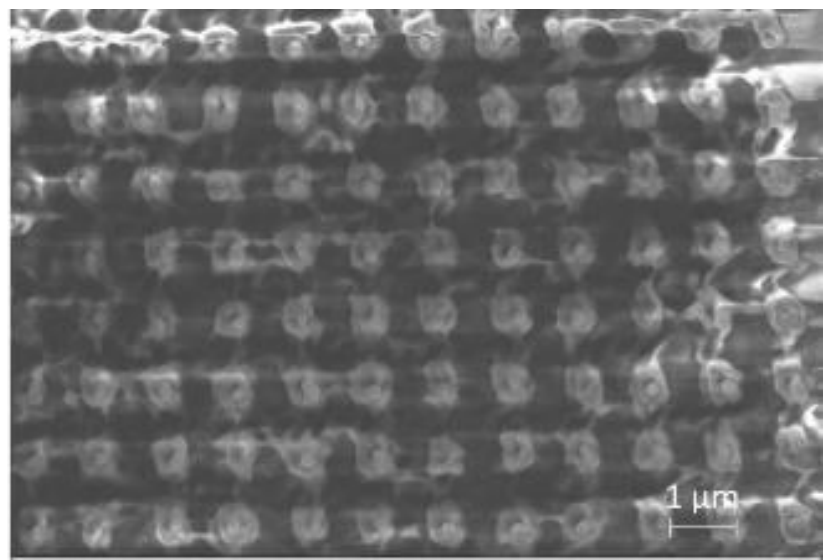

Fig. 4. SEM image of a nanostructure array.

\section{RESULTS}

After HTPP fabrication, the nanostructures were investigated using SEM. In the SEM image, the $8 \times 12$ periodic nanostructures were a part of the $25 \times 25$ periodic nanostructures. Each nanostructure was integrated with five voxels, which were clearly observed in the SEM image; nanolines between nanostructures were generated in overlapping weakly polymerized regions. As shown in Fig. 4, the distance between nanostructures was $1 \mu \mathrm{m}$, and each nanostructure was approximately $630 \pm 55 \mathrm{~nm}$ wide.

The light distributions shown in Fig. 5(a) and Fig. 5(b) were measured using white-light 3D microscopy, and the light distributions shown in Fig. 5(c) and (d) were simulated using COMSOL at a 545-nm wavelength. As shown in Fig. 5, the 
glass surface was located at the $0-\mu \mathrm{m}$ position, the air and structure were above the glass surface, and the glass substrate was below the glass surface. The slice images shown in Fig. 5(a) and Fig. 5(b) were taken using a CMOS camera, the range of the scanning area above the glass surface was $5 \mu \mathrm{m}$ with a $0.2-\mu \mathrm{m}$ interval and that below the glass surface was 5 $\mu \mathrm{m}$ with a $0.2-\mu \mathrm{m}$ interval; the camera was attached to a moving piezo stage. For image processing, the whole slice images were reconstructed using ImageJ software to generate a 3D image. Fig. 5(a) shows a side view of the 3D image. Fig. 5(b) depicts the image in the green channel only, which is different from the color channels of the image shown in Fig. 5 (a); for the image shown in the green channel, the scanning extent was restricted to $2 \mu \mathrm{m}$ above and $2 \mu \mathrm{m}$ below the glass surface. However, Fig. 5(c) and Fig. 5(d) were simulated using COMSOL software. The light distribution was obtained using the nanostructures that were $640 \mathrm{~nm}$ wide and $1000 \mathrm{~nm}$ high with a refractive index of 1.47 . The image depicted in Fig. 5(c) was cropped from that shown in Fig. 5(d), and the extent of the cropped image was restricted to $2 \mu \mathrm{m}$ above the glass surface and $2 \mu \mathrm{m}$ below the glass surface. Comparing Fig. 5(b) with Fig. 5(c) indicates that the first central hot spot between nanostructures was located at approximately $910 \pm$ $50 \mathrm{~nm}$ and $890 \mathrm{~nm}$. In other words, the experimental central hot spot position was corresponded with the simulated position. The results of the light distribution experiment agreed with those of the simulation.

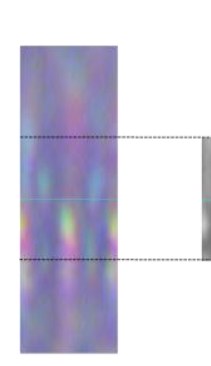

(a)

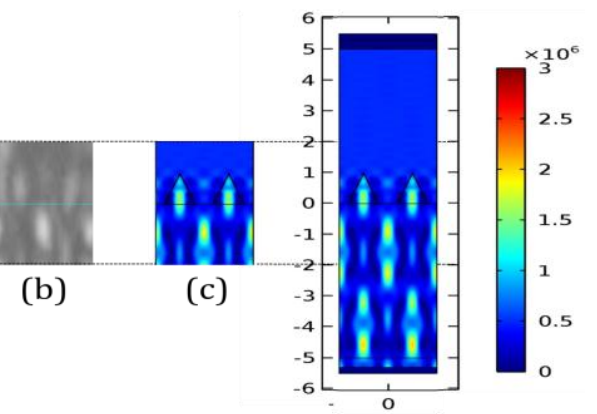

(d)
Fig. 5. 3D light distribution above (reflection) and below the nanostructure array on the glass substrate. (a) Measured using 3D microscopy, (b) image cropped from that in (a) at a extent of $2 \mu \mathrm{m}$ above the glass surface and $2 \mu \mathrm{m}$ below the glass surface only the green channel, (c) image cropped from that in (d) at an extent of $2 \mu \mathrm{m}$ above and $2 \mu \mathrm{m}$ below the glass surface, and (d) image simulated using COMSOL at a $545-\mathrm{nm}$ wavelength.

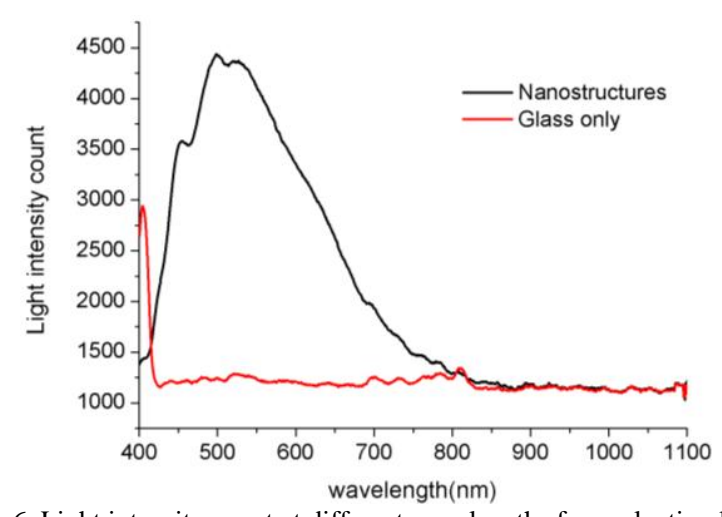

Fig. 6. Light intensity count at different wavelengths for evaluating light extraction capability of nanostructures and glass only.

After the light distribution experiment, we used electroless gold plating technology to coat the gold layer on the nanostructures only. As shown in Fig. 6, the light intensity counts for evaluating the light extraction capability were measured using the spectrometer. The highest peak of the light intensity counts was 4436.2 at a $498.54-n m$ wavelength. At the same wavelength, the light intensity of the glass only (without nanostructures) was 1247.1. The light extraction capability of the nanostructures was 3.6 times higher than that of the glass only.

\section{CONCLUSION}

In this study, we demonstrated HTPP fabrication of nanostructures at a rate of $\mathrm{mm}^{2}$ per minute by using $25 \times 25$ hologram laser splitting. The results of the simulation and experimental 3D light distribution induced by the 3D nanostructures are in favorable agreement. However, the light extraction capability of the nanostructures is 3.6 times higher than that of the glass substrate only. Such high-speed fabrication of complex 3D nanostructures can be useful for applications such as light extraction or absorption in thin films or for metamaterial surfaces after electroless gold plating. Future study will entail optimizing the design of voxel arrangements for increasing light extraction efficiency in various optical surface applications.

\section{REFERENCES}

[1] H. H. Pham, I. Gourevich, J. K. Oh, J. E. Jonkman, and E. Kumacheva, "A multidye nanostructured material for optical data storage and security data encryption," Advanced Materials, vol. 16, no. 6, pp. 516-520, 2004.

[2] D. J. Lipomi, R. V. Martinez, M. A. Kats, S. H. Kang, P. Kim, J. Aizenberg, F. Capasso, and G. M. Whitesides, "Patterning the tips of optical fibers with metallic nanostructures using nanoskiving," Nano Letters, vol. 11, no. 2, pp. 632-636, 2010.

[3] C. Paquet and E. Kumacheva, "Nanostructured polymers for photonics," Materials Today, vol. 11, no. 4, pp. 48-56, 2008.

[4] M. L. Brongersma, Y. Cui, and S. Fan, "Light management for photovoltaics using high-index nanostructures," Nature Materials, vol. 13, no. 5, pp. 451-460, 2014.

[5] H. Misawa, T. Kondo, S. Juodkazis, V. Mizeikis, and S. Matsuo, "Holographic lithography of periodic two-and three-dimensional microstructures in photoresist SU-8," Optics Express, vol. 14, no. 17, pp. 7943-7953, 2006.

[6] S. Rekštytė, A. Žukauskas, V. Purlys, Y. Gordienko, and M. Malinauskas, "Direct laser writing of 3D polymer micro/nanostructures on metallic surfaces," Applied Surface Science, vol. 270, pp. 382-387, 2013.

[7] B. B. Xu, Y. L. Zhang, H. Xia, W. F. Dong, H. Ding, and H. B. Sun, "Fabrication and multifunction integration of microfluidic chips by femtosecond laser direct writing," Lab on a Chip, vol. 13, no. 9, pp. 1677-1690, 2013.

[8] T. L. Chang, Z. C. Chen, and Y. C. Lee, "Micro/nano structures induced by femtosecond laser to enhance light extraction of GaN-based LEDs," Optics Express, vol. 14, no. 17, pp. 15997-16001, 2012.

[9] Y. J. Jeong, T. W. Lim, Y. Son, D. Y. Yang, H. J. Kong, and K. S. Lee, "Proportional enlargement of movement by using an optically driven multi-link system with an elastic joint," Optics Express, vol. 18, no. 13, pp. 13745-13753, 2010.

[10] S. J. Maruo and H. Inoue, "Optically driven micropump produced by three-dimensional two-photon microfabrication," Applied Physics Letters, vol. 89, no. 14, pp. 144101-144103, 2006.

[11] F. Jipa, M. Zamfirescu, A. Velea, M. Popescu, and R. Dabu, Femtosecond Laser Lithography in Organic and Non-Organic Materials, Updates in Advanced Lithography, 2013, ch. 3.

[12] F. Formanek, N. Takeyasu, T. Tanaka, K. Chiyoda, A. Ishikawa, and S. Kawata, "Three-dimensional fabrication of metallic nanostructures over large areas by two-photon polymerization," Optics Express, vol. 14, no. 2, pp. 800-809, 2006. 
[13] B. Chichkov, "Two-photon polymerization enhances rapid prototyping of medical devices," SPIE Newsroom, vol. 10, pp. 1-2, 2007.

[14] S. D. Gittard, A. Nguyen, K. Obata, A. Koroleva, R. J. Narayan, and B. N. Chichkov, "Fabrication of microscale medical devices by two-photon polymerization with multiple foci via a spatial light modulator," Biomedical Optics Express, vol. 2, no. 11, pp. 3167-3178, 2011.

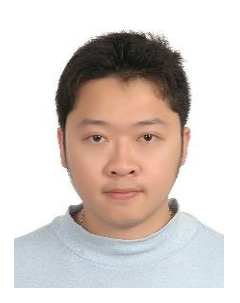

Yi-Hsiung Lee received his B.S. degree in automatic control engineering from Feng Chia University in 2010. After receiving his B.S. degree, he directly studied his Ph.D. with the Prof. Yi-Jui Liu in the Program of Electrical and Communications Engineering, Feng Chia University, Taiwan. Since 2010, he joined the group of Prof. Chih-Lang Lin in the Central Taiwan University of Science and Technology, Taiwan. In 2014, He earned the "Eiffel doctoral scholarships" in France and worked with the Prof. Patrice L. Baldeck in Ecole Normale Superieure de Lyon, France. His research interests include micro- and nanomachine applications, image processing. He proposed a serious of mechanical elementals, such as spring, lever beam, gears, Archimedes screw, etc., fabricated by two-photon polymerization and flexible driven by optical tweezers. More recently, he is studying the periodic 3D nanostructuration which was fabricated by holographic two-photon polymerization for contributing to optical surfaces.

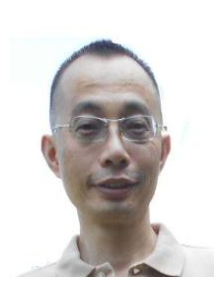

Yi-Jui Liu received his Ph.D. degree in electric engineering from National Taiwan University in 2002. From 2002 to 2004, he was an assistant professor in the Department of Medical Imaging and Radiological Technology at Yuan Pei University in Taiwan. From 2004 until now, he is a teacher in the Department of Automatic Control Engineering at Feng Chia University in Taiwan. He is currently a professor of biomedical engineering and expertise in magnetic resonance imaging, biomedical image processing and biomedical signal processing. His main research activity is in the change of blood velocity and BOLD signal by carbon dioxide. Since 2012, he cooperated with Prof. Chih-Lang Lin in the Central Taiwan University of Science and Technology, Taiwan. Their research interests include micro/nanomachine applications and image processing. More recently, they are studying the fabrication of 3D structured protein by using two-photon polymerization technology for detecting bio-cells such as bacteria, red blood cells, and cancer cells, and the bio-mechanics of cells by using optical tweezers for the clinical diagnosis. All of the above topics are studied for contributing to Lab-on-a-chips.

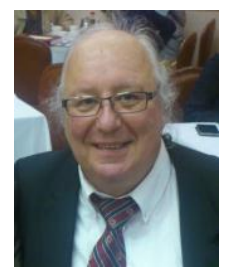

Patrice L. Baldeck graduated in physics from the University Pierre and Marie Curie (Paris VI), 1983, received the master degree in microwave and optoelectronics from University Pierre and Marie Curie (Paris VI), 1985, and the Ph.D. in electrical engineering/photonics from the EE department of City College of New York, 1989, with postdoc at Laboratory of Photo Physics and Photochemistry CEA-Saclay. He was the CNRS research professor at Laboratory of Interdisciplinary Physics at Grenoble University, from 1989 to 2015, and the CNRS research professor at Laboratory of Chemistry at ENS-Lyon, since 2011. He was an honorary chair professor in bio-photonics at Central Taiwan University of Science and Technology, from 2011 to 2015. He has strong research collaborations with international universities in Taiwan, Romania, Spain, Algeria, and Brazil, and has experience in ultrafast lasers, nonlinear optics, molecular spectroscopy, plasmonics, and biophotonics. His current research activity concerns the development of new laser applications with high spatial selectivity, based on the two-photon absorption properties of molecules at the focal point of lasers: micro/nano 3D printing, bioimaging and phototherapy. He has been the leader, or principal investigator of 26 funded research projects for a total funding of $106 €$. He has tutored $23 \mathrm{PhD}$ students, 7 post-docs, and more than 70 master students. He is the coauthor of 138 publications in international journals, 50 conference proceedings, and 5 book chapters. He holds 3 patents. He has presented or co-authored 81 invited lectures in international conferences.

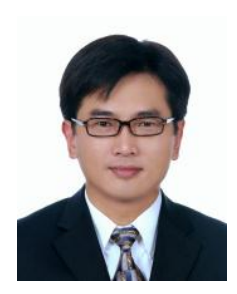

Chih-Lang Lin received his master's degree in power mechanical engineering from National Tsing-Hua University. He earned his Ph.D. degree in mechanical engineering from National Taiwan University. In the meanwhile, he earned another Ph.D. degree in physics of condensed material and radiation from Joseph Fourier University, France. His thesis involves two topics which are fiber Bragg grating (FBG) sensors and laser driven microsensors. In the first part, a framework for the interpretation of reflected FBG spectra under a non-uniform strain field is proposed and experimental results for a crack tip strain field are presented. In the second part, the fabrication of laser driven polymer microsensors for visconsimetry, velocimetry and micropump applications are developed. Before he created the Bio-Photonics Lab at Central Taiwan University of Science and Technology, he joined Air Liquide international group and worked in Japan to be a researcher and in Taiwan to be an operation manager. One of Dr. Lin's research interests is laser driven micromachines. He proposed a serious of elemental micromachines, such as cantilever, lever beam, spring, Archimedes screw, etc. His another interest is the fabrication of three-dimensional structured protein by using two-photon polymerization technology for detecting bio-cells such as bacteria, red blood cells, and cancer cells. More recently, he is studying the bio-mechanics of cells by using optical tweezers for the clinical diagnosis. All of the above topics are studied for contributing to Lab-on-a-chips. 
Electronic and Communication Engineering 
